\title{
The functional prediction of transmembrane serine protease 2 (TMPRSS2) in priming S-protein of SARS-CoV-2 among vertebrates
}

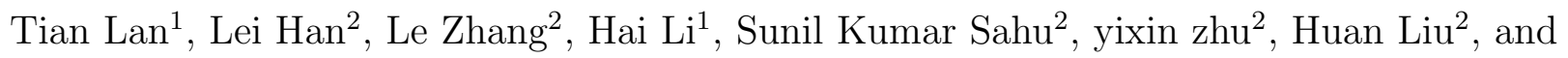
Yan $\mathrm{Hua}^{2}$

${ }^{1}$ BGI-Shenzhen

${ }^{2}$ Affiliation not available

June 15,2020

\begin{abstract}
The coronavirus disease 19 (COVID-19) is caused by severe acute respiratory syndrome coronavirus 2 (SARS-CoV-2), and the outbreak has rapidly spread worldwide causing a global severe pneumonia pandemic. Several animals have been proven to be affected by the SARS-CoV-2, posing an enormous threat to humans. However, to confirm the intermediate hosts in the epidemic regions, it is unrealistic to detect its presence in each and every animal one by one. An active transmembrane serine protease 2 (TMPRSS2) which functions in priming of S-protein of SARS-CoV-2 is one of the most important prerequisites for an animal to be an intermediate host, but the utilizing capability of TMPRSS2 for non-human animals is largely unknown. Here, we systematically predicted the ability of TMPRSS2 to prime S-protein of SARS-CoV-2 in 164 vertebrates by analyzing the phylogenetic clustering and possible functional changes induced by amino acid variation in TMPRSS2. Mammals were predicted to be the most likely animals to effectively use the TMPRSS2 for priming S-protein of SARS-CoV-2. Especially, we predicted the possible effective utilizing ability of TMPRSS2 in rats and pigs, which are reported non-susceptible to SARS-CoV-2. Given the wide distribution and the close relationships among human, pig and rat, a periodic and careful monitoring is required for pigs and rats to prevent the possible SARS-CoV-2 transmission to human.
\end{abstract}

The functional prediction of transmembrane serine protease 2 (TMPRSS2) in priming S-protein of SARSCoV-2 among vertebrates

Tianming Lan ${ }^{1,3^{*}}$, Han Lei ${ }^{1} 5^{*}$, Le Zhang ${ }^{1,5^{*}}$, Haimeng $\mathrm{Li}^{4}$, Sunil Kumar Sahu ${ }^{1,6}$, Yixin Zhu ${ }^{4}$, Huan Liu ${ }^{7}$, Yan $\mathrm{Hua}^{2 \#}$

1. BGI-Shenzhen, Shenzhen 518083, China

2. Guangdong Provincial Key Laboratory of Silviculture, Protection and Utilization, Guangdong Academy of Forestry, Guangzhou 510520, China

3. Department of Biology, University of Copenhagen, DK-2100 Copenhagen, Denmark

4. School of Future Technology, University of Chinese Academy of Sciences, Beijing 100049, China

5. College of Wildlife And Protected Area, Northeast Forestry University. Harbin 150040, China Guangdong Provincial Academician Workstation of BGI Synthetic Genomics,BGI-Shenzhen, Shenzhen, 518120,China

6. State Key Laboratory of Agricultural Genomics, BGI-Shenzhen, Shenzhen 518083, China

7. Guangdong Provincial Key Laboratory of Genome Read and Write, BGI-Shenzhen 518083, Shenzhen, China

*These authors contributed equally 
\# Correspondence should be addressed to: Yan Hua (wildlife530@hotmail.com)

Abstract

The coronavirus disease 19 (COVID-19) is caused by severe acute respiratory syndrome coronavirus 2 (SARSCoV-2), and the outbreak has rapidly spread worldwide causing a global severe pneumonia pandemic. Several animals have been proven to be affected by the SARS-CoV-2, posing an enormous threat to humans. However, to confirm the intermediate hosts in the epidemic regions, it is unrealistic to detect its presence in each and every animal one by one. An active transmembrane serine protease 2 (TMPRSS2) which functions in priming of S-protein of SARS-CoV-2 is one of the most important prerequisites for an animal to be an intermediate host, but the utilizing capability of TMPRSS2 for non-human animals is largely unknown. Here, we systematically predicted the ability of TMPRSS2 to prime S-protein of SARS-CoV-2 in 164 vertebrates by analyzing the phylogenetic clustering and possible functional changes induced by amino acid variation in TMPRSS2. Mammals were predicted to be the most likely animals to effectively use the TMPRSS2 for priming S-protein of SARS-CoV-2. Especially, we predicted the possible effective utilizing ability of TMPRSS2 in rats and pigs, which are reported non-susceptible to SARS-CoV-2. Given the wide distribution and the close relationships among human, pig and rat, a periodic and careful monitoring is required for pigs and rats to prevent the possible SARS-CoV-2 transmission to human.

\section{Introduction}

The COVID-19 has been rapidly spreading worldwide since its outbreak during late 2019[1]. Now it has become a global pandemic with 7,039,918 cases and 404,396 deaths globally as of 9 June 2020[2]. The COVID19 usually causes severe respiratory illness and transmitted human-to-human, like severe acute respiratory syndrome (SARS) and the Middle East respiratory syndrome (MERS), which has been proved to be caused by SARS-CoV-2[3]. At present, no effective vaccines and drugs have been developed to prevent or cure the infection of SARS-CoV-2.

A $96.2 \%$ whole genome sequence identity between the human SARS-CoV-2 and the bat (Rhinolophus affinis ) coronavirus Bat-CoV-RaTG13 indicated the probable bat origin of the SARS-CoV-2[4]. However, the SARS-CoV-2 is less likely to transfer to humans from bats because of the lack of direct contact between humans and bats. Similar to SARS-CoV, the intermediate hosts are also expected to serve as bridges for transmission of SARS-CoV2 between humans and animals. To date, several animals have been found to be potential intermediate hosts, including pangolins, dogs, cats, ferrets and hamsters $[5,6,7,8,9,10,11]$. A metallopeptidase, angiotensin converting enzyme II (ACE2) has been proven to be the cell entry receptor of SARS-CoV[12]. Similarly, SARS-CoV-2 also uses the ACE2 as a cognate receptor to enter into target cells[4]. The initial interaction between the S-protein of SARS-CoV-2 and ACE2 help SARS-CoV-2 attach to target cells. In addition, cell entry requires S-protein priming by cellular proteases, the TMPRSS2, which can activate and cleave the S-protein and facilitate the membrane fusion of virus and cell[13]. ACE2 and TMPRSS2 are supposed to be indispensable for SARS-CoV-2 infection.

Identifying possible intermediate hosts can directly contribute to the prevention and control for the transmission of SARS-CoV-2. As COVID-19 is a worldwide pandemic now, it is difficult to detect SARS-CoV-2 in animals which are distributed all across the world to determine the hosts. Phylogenetic analysis and critical site comparison of ACE2 has been used for predicting the intermediate hosts of SARS-CoV-2, showing that mammals,pangolins and cats are supposed to be more susceptible to SARS-CoV-2 than birds[14]. However, it is to be noted that the SARS-CoV-2 cannot enter into target cells without S-protein priming mediated by TMPRSS2. In this study, we combined sequence alignment, phylogenetic analysis and potential damaging effects on amino acid changes in TMPRSS2 to systematically predict the most likely intermediate hosts by screening 164 species from the perspective of TMPRSS2's function on S-protein priming of SARS-CoV-2.

\section{Material and Methods}

\subsection{Samples and data}

164 complete TMPRSS2 protein sequences of 164 animal species (one protein sequence for each species) were 
collected from NCBI, including 80 Mammalia, 34 Aves, 13 Reptilia, 3 Amphibia, and 34 fish. Pig, horse, cattle, duck, goose, chicken, dog, cat, and hamster that have potentially direct contacts with humans.. The accession number of each sequence is listed in Table S1.

\subsection{Phylogenetic analysis}

The phylogenetic tree of all 164 species and 38 selected species was constructed by MEGA X[15] using the neighbor-joining (NJ) method with 1000 bootstrap replicates. Multiple sequences alignment used for phylogenetic tree construction was generated by the MAFFT software[16] with default parameters. We further searched for conserved domains within human TMPRSS2 protein against the NCBI's CDD v3.18 - 55570 PSSMs database[17] by setting the expected value threshold of 0.01 and 500 maximum number of hits. We then constructed a phylogenetic tree using protein sequences of domain regions by use of the same NJ method as the whole amino sequences.

\subsection{Predicting the impact of amino acid substitutions on the function of TMPRSS2}

The human TMPRSS2 protein sequence was selected as the reference to screen all amino changes across 164 species. The impact of an amino acid change on the structure and function of the TMPRSS2 was finally estimated by the PolyPhen-2[18]. Scores were calculated by PolyPhen-2 for estimating the effects of amino acid changes on the protein, and were divided into three groups: probably damaging (score [?] 0.957), possibly damaging $(0.453<$ score $<0.956)$ and benign (score [?] 0.452). To facilitate the prediction of capability of TMPRSS2 for S-protein priming, we marked each species based on the PolyPhen-2 scores. We only focused on domain regions. Firstly, we considered the full mark as 100, as the human TMPRSS2 protein sequence has been proved to work in human cells. Secondly, if amino acid changes was not found to occur at catalytic triad; $0.1,0.5$ and 1 point deduction was made for "benign", "possibly damaging" and "probably damaging", respectively. Thirdly, if one amino acid change occurred at the catalytic triad of TMPRSS2 (His296, Ser441 and Asp435), 15 points deduction was made for three reasons: 1) the change of catalytic triad greatly reduced the enzyme activity; 2) the poultry were proven much less susceptible to SARS-CoV-2; 3) scores of all Aves were less than 85. Finally, we ranked scores for all species to predict the potential function of TMPRSS2 for S-protein priming of SARS-CoV-2.

3 Results

\subsection{Phylogenetic relationship of TMPRSS2 among 164 vertebrates}

A phylogenetic tree consisting of 164 vertebrate species were constructed using the whole protein sequence of TMPRSS2 to evaluate the homology across species (Fig S1). The tree showed four main clades. The Chondrichthyes TMPRSS2 formed the most basal branch, followed bt the Osteichthyes and Amphibia TMPRSS2 clades. The TMPRSS2 of Mammalia, Reptilia, and Aves formed the last clade, in which the Mammalia TMPRSS2 formed the sister cluster with that of Reptilia and Aves. The phylogenetic relationship of TMPRSS2 among different species was highly consistent with the evolutionary relationships at the species level, as well as the genetic distance (Table S2). We further confirmed the start and end positions of three main conserved domain regions of TMPRSS2 to focus on this analysis in more conserved functional regions (Fig 1, Fig S2, Fig S3). The overall structure of the tree built with the conserved region was well in line with the tree constructed using the complete sequences with only a few detailed differences. We further built a phylogenetic tree of TMPRSS2 using selected species that are closer to humans (Fig 2). Important livestock, like pig, sheep and cattle, was closely clustered with cat, dog, and pangolin, which are shown to be effected by SARS-CoV-2. The horse was also placed with the human. In addition, rat, mouse and hamster shared the same clade with human. Bird, reptile, amphibian and fish were not found to be mixed into the susceptible animals. 


\section{A}

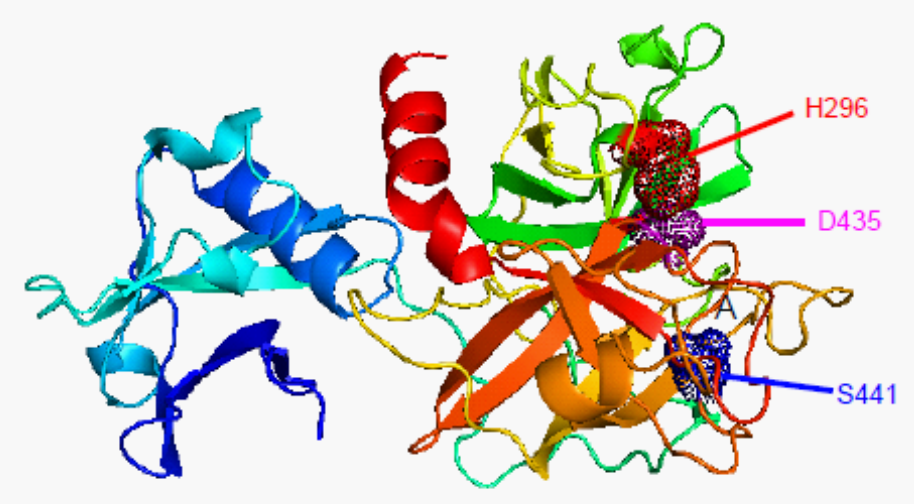

B

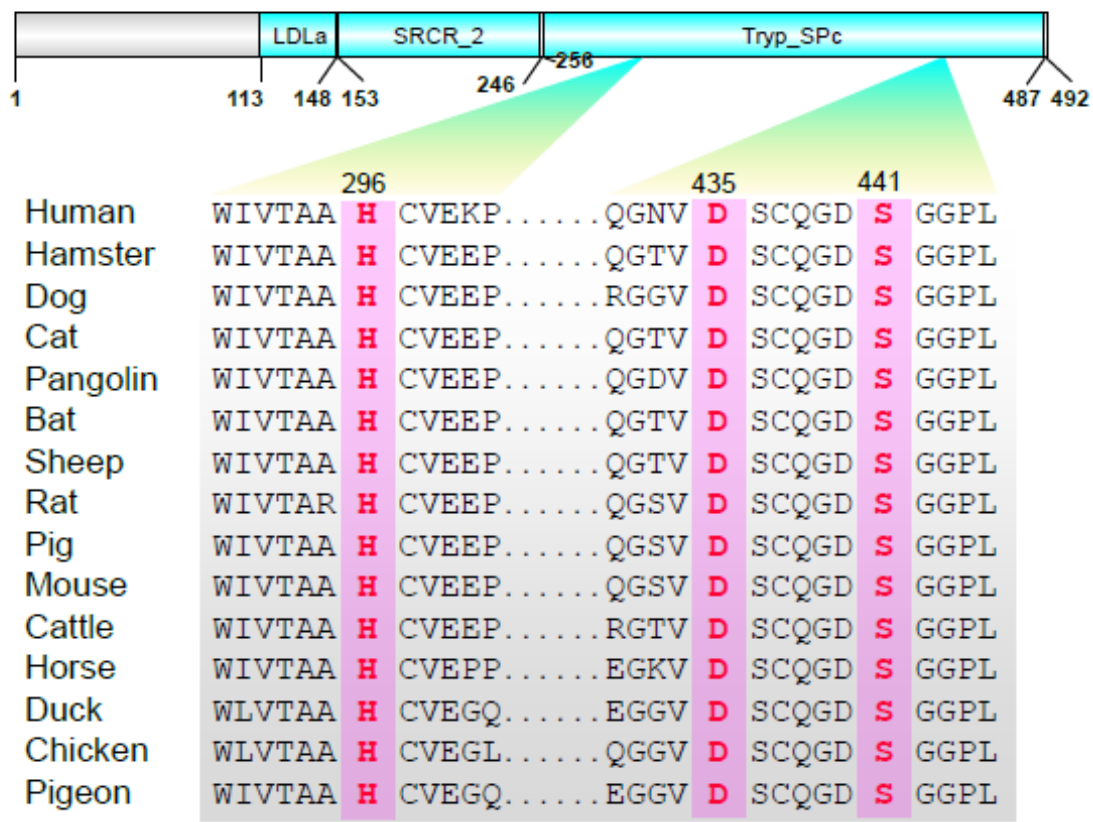

Fig 1. The conserved domain regions and the catalytic triad of TMPRSS2 in selected animals. A. The protein secondary structure of TMPRSS2 predicted by SWISS-MODEL[19] and the catalytic triad amines. B. The TMPRSS2 has three conserved domain regions: LDLa, SRCR_2 and Tryp_SPc. Three catalytic triad H296, S435 and D441 were ultra-conserved across species. 


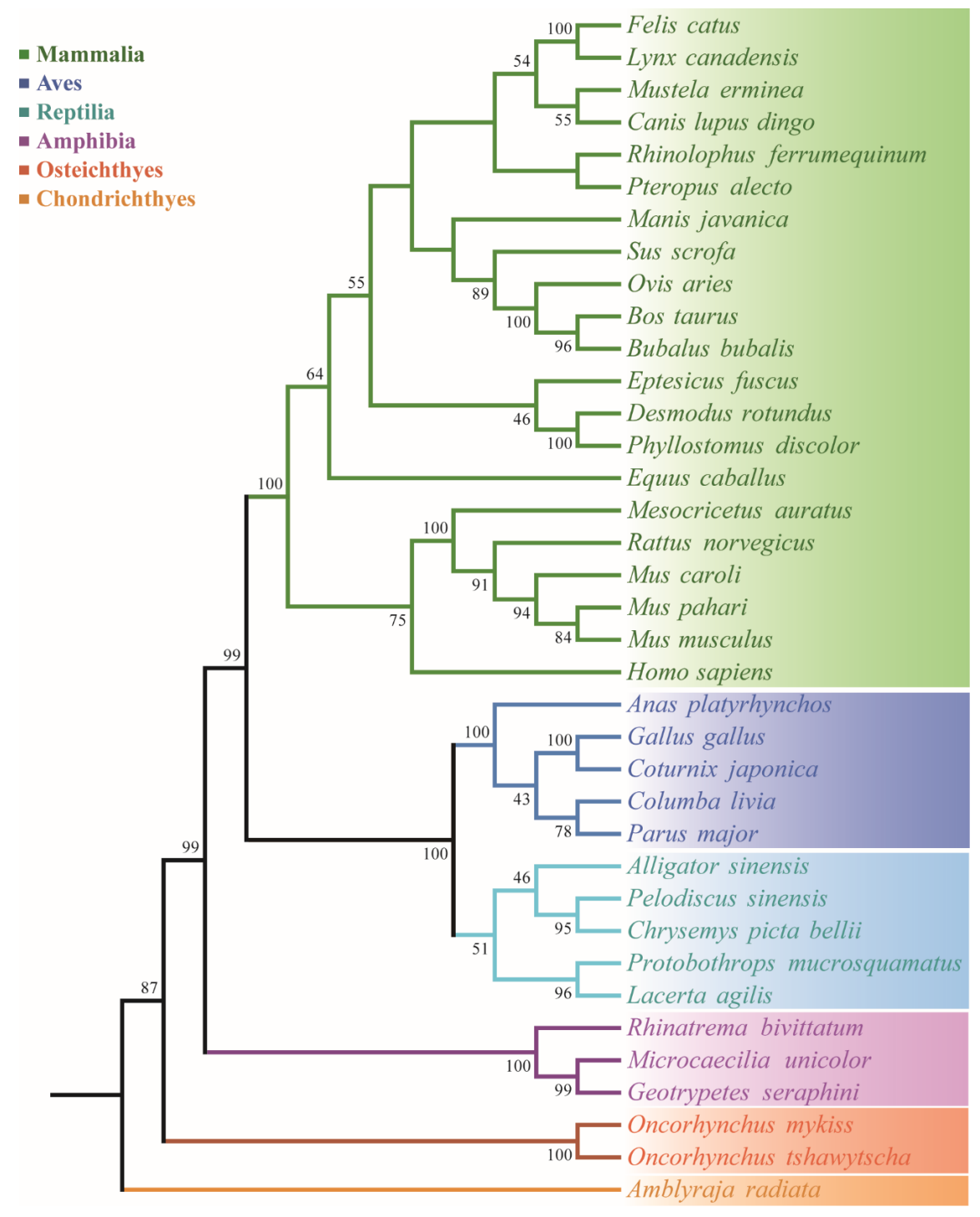

Fig 2. The phylogenetic tree of TMPRSS2 from selected species. The tree was constructed using the NJ method (1000 bootstraps) implanted in the MEGA X based on whole amino sequences of TMPRSS2.

\subsection{Prediction of functional constraints of the TMPRSS2 across vertebrate species}

We scored the TMPRSS2 protein sequence for all the collected 164 species (Table $1 \&$ Table S3). Scores from high to low were correspondent to Mammalia, Aves, Reptilia, Amphibia, Osteichthyes and Chondrichthyes, except for the Puma concolor, Athene cunicularia, Geospiza fortisand Anarrhichthys ocellatus, because these four species have amino change at the catalytic triad of TMPRSS2. From the global point of view, this result was highly consistent with the prediction from the phylogenetic tree. As expected, the primates scored the highest marks of over 95. Besides, other animals have been proven to be infected with the SARS-CoV-2, including pangolin, cat, dog, and hamster, have scored higher than 91. Especially, no damaging amino acid change was predicted in the hamster. In addition, only one probably or possibly damaging mutation was found in rat and mouse, even lesser than that of pangolin and cat. When focusing on animals that are closely related to humans, livestock has a much higher score than that of poultry.

Table 1. Prediction of functional constraints of the TMPRSS2 in 36 selected species. 


\begin{tabular}{|c|c|c|c|}
\hline Species & Number of amino acid changes & Number of amino acid changes & Number of \\
\hline & Benign & Possibly damaging & Probably d \\
\hline Rhinolophus ferrumequinum & 55 & 1 & 0 \\
\hline Canis lupus dingo & 58 & 1 & 0 \\
\hline Ovis aries & 59 & 1 & 0 \\
\hline Mus pahari & 60 & 1 & 0 \\
\hline Bos taurus & 63 & 1 & 0 \\
\hline Mesocricetus auratus & 68 & 0 & 0 \\
\hline Bubalus bubalis & 64 & 1 & 0 \\
\hline Eptesicus fuscus & 65 & 1 & 0 \\
\hline Rattus norvegicus & 66 & 1 & 0 \\
\hline Mus musculus & 63 & 0 & 1 \\
\hline Sus scrofa & 64 & 2 & 0 \\
\hline Manis javanica & 64 & 3 & 0 \\
\hline Pteropus alecto & 61 & 2 & 1 \\
\hline Felis catus & 59 & 3 & 1 \\
\hline Mustela erminea & 61 & 3 & 1 \\
\hline Lynx canadensis & 62 & 3 & 1 \\
\hline Equus caballus & 76 & 3 & 0 \\
\hline Desmodus rotundus & 67 & 3 & 3 \\
\hline Phyllostomus discolor & 65 & 5 & 3 \\
\hline Gallus gallus & 125 & 4 & 0 \\
\hline Columba livia & 110 & 7 & 1 \\
\hline Coturnix japonica & 120 & 7 & 1 \\
\hline Anas platyrhynchos & 127 & 8 & 0 \\
\hline Parus major & 122 & 8 & 1 \\
\hline Chrysemys picta bellii & 98 & 11 & 4 \\
\hline Pelodiscus sinensis & 104 & 12 & 3 \\
\hline Alligator sinensis & 112 & 16 & 1 \\
\hline Protobothrops mucrosquamatus & 117 & 17 & 7 \\
\hline Lacerta agilis & 109 & 21 & 8 \\
\hline Rhinatrema bivittatum & 121 & 19 & 10 \\
\hline Microcaecilia unicolor & 121 & 23 & 8 \\
\hline Geotrypetes seraphini & 113 & 22 & 14 \\
\hline Oncorhynchus tshawytscha & 134 & 26 & 10 \\
\hline Oncorhynchus mykiss & 135 & 26 & 10 \\
\hline Mus caroli & 51 & 6 & 20 \\
\hline Amblyraja radiata & 120 & 34 & 35 \\
\hline
\end{tabular}

\section{Discussion}

Overall, Mammalia, Aves, and Reptilia formed a distinct cluster, indicating the TMPRSS2 was more likely to be used for recognizing and processing S-protein of SARS-CoV-2 in Aves and Reptilia rather than Amphibia and fish. Human, pangolin, cat, dog and hamster are proved to be be infected by SARS-CoV-2 in previous studies $[5,6,7,8,9,10,11]$, especially for human, the TMPRSS2 was shown to be used for priming S-protein on ACE2 to help SARS-CoV-2 to enter into cells[13]. We then focused on these species to predict the utilizing capability of TMPRSS2 for S-protein priming. Primates closely clustered together with human (Fig S1, Fig S2), indicating the TMPRSS2 could be effectively used in primates for S-protein priming. Cat, dog clustered with bat (Fig 2), which was inconsistent with the phylogenetic result of ACE2[14], and also showed the high possibility of TMPRSS2 utilizing capability. The brown bear was placed with pangolin, bat and dog forming a distinct clade (Fig S1), showing yet another possible wild animal of high probability to use TMPRSS2 to 
cleave S-protein of SARS-CoV-2. Considering the lack of direct contact with the human, brown bear was less likely to be the intermediate host to transfer the virus to human. Interestingly, the TMPRSS2 of horse closely clustered with hamster, but not with its much closer relatives (Fig S1), showing a potentially effective function of TMPRSS2 in horse to process the S-protein for priming. When focused on animals that have a close relationship with humans; the pig, sheep, cattle and pangolin formed a distinct clade (Fig 2). Although the incubation test showed the pig was not susceptible with SARS-CoV-2 infection[7], our result indicated the probable availability of TMPRSS2 for S-protein priming in the pig. A previous study showed that the SARS-CoV-2 was not able to use ACE2 to enter into the mouse cell[4]. In our study, however, rat and mouse TMPRSS2 were closely clustered with human and hamster TMPRSS2, showing that the TMPRSS2 in mouse and rat should be able to activate the S-protein of SARS-CoV-2.

ACE2 can tolerate a variety of amino acid changes among animals, making the low species barrier of SARSCoV-2[20]. We expected the same situation for TMPRSS2, so we further used the PolyPhen-2 to evaluate the possible impact on the function of TMPRSS2 at single amino level, but not at the whole sequence level, because many amino acid changes will not cause change in function. By scoring the TMPRSS2 of all 164 species, we found that the prediction result was highly similar to prediction from the phylogenetic tree, with Mammalia having the highest scores, and then Aves, Reptilia, Amphibia and fish. However, the most important three amines (His296, Ser441 and Asp435)[21] located at the catalytic triad of TMPRSS2 were ultra-conserved (Fig 1, Fig S4). Only four out of 164 species were found to have mutations at these three amines, indicating a conserved utilizing capability of TMPRSS2 across species, although other amino acid changes may also influence the efficiency of TMPRSS2 to cleave the S-protein of SARS-CoV-2. Animals that have been proven to be affected by the SARS-CoV-2, like pangolin, cat, dog, and hamster, exhibited very high scores (Table 1), which showed the rationality of this scoring method. Both high score and less damaging amino acid changes were found in rat, mouse and pig than pangolin and cat, which in turn supported the prediction of the phylogenetic tree, showing the effective utilizing capability of TMPRSS2 in rat, mouse and pig. In addition, both the phylogenetic tree and PolyPhen-2 scores showed relatively low TMPRSS2 capability in poultry.

COVID19 is still progressing and SARS-COV-2 strains are constantly evolving. It needs to be emphasized again that we need to pay more attention to mammals, especially pigs and rats, to prevent these animals from becoming intermediate hosts of a future pandemic, considering the more effective utilizing capability of TMPRSS2 in mammals than in other animals.

\section{Author Contribution}

YH conceived and designed the project. TML and LH were responsible for collecting the data. TML coordinated the data analysis. LH, and LZ contributed to data analysis. HML, and YXZ participated in data interpretation and visualization. TML and SKS wrote the manuscript.

\section{Competing interest}

The authors declare no competing interests.

\section{Data accessibility statement}

All data generated or analyzed during this study are included in this article and Supplementary materials. All the authors agree to deposit data to public repository.

\section{Acknowledgement}

This work was supported by China National GeneBank (CNGB). Our project was financially supported by funding from the Guangdong Provincial Key Laboratory of Genome Read and Write (grant No. 2017B030301011). We thank the Guangdong Provincial Academician Workstation of BGI Synthetic Genomics (No. 2017B090904014).

[1] L. The, Emerging understandings of 2019-nCoV, The Lancet 395 (2020) 311. 
[2] World Health Organization. WHO Novel Coronavirus (2019-nCoV) situation reports, https:// www.who.int/emergencies/diseases/novel-coronavirus-2019/situation-reports/.

[3] N. Zhu, D. Zhang, W. Wang, X. Li, B. Yang, J. Song, X. Zhao, B. Huang, W. Shi, R. Lu, P. Niu, F. Zhan, X. Ma, D. Wang, W. Xu, G. Wu, G.F. Gao, W. Tan, I. China Novel Coronavirus, T. Research, A Novel Coronavirus from Patients with Pneumonia in China, 2019, N Engl J Med 382 (2020) 727-733.

[4] P. Zhou, X.L. Yang, X.G. Wang, B. Hu, L. Zhang, W. Zhang, H.R. Si, Y. Zhu, B. Li, C.L. Huang, H.D. Chen, J. Chen, Y. Luo, H. Guo, R.D. Jiang, M.Q. Liu, Y. Chen, X.R. Shen, X. Wang, X.S. Zheng, K. Zhao, Q.J. Chen, F. Deng, L.L. Liu, B. Yan, F.X. Zhan, Y.Y. Wang, G.F. Xiao, Z.L. Shi, A pneumonia outbreak associated with a new coronavirus of probable bat origin, Nature 579 (2020) 270-273.

[5] T.T. Lam, M.H. Shum, H.C. Zhu, Y.G. Tong, X.B. Ni, Y.S. Liao, W. Wei, W.Y. Cheung, W.J. Li, L.F. Li, G.M. Leung, E.C. Holmes, Y.L. Hu, Y. Guan, Identifying SARS-CoV-2 related coronaviruses in Malayan pangolins, Nature (2020).

[6] K. Xiao, J. Zhai, Y. Feng, N. Zhou, X. Zhang, J.J. Zou, N. Li, Y. Guo, X. Li, X. Shen, Z. Zhang, F. Shu, W. Huang, Y. Li, Z. Zhang, R.A. Chen, Y.J. Wu, S.M. Peng, M. Huang, W.J. Xie, Q.H. Cai, F.H. Hou, W. Chen, L. Xiao, Y. Shen, Isolation of SARS-CoV-2-related coronavirus from Malayan pangolins, Nature (2020).

[7] J. Shi, Z. Wen, G. Zhong, H. Yang, C. Wang, B. Huang, R. Liu, X. He, L. Shuai, Z. Sun, Y. Zhao, P. Liu, L. Liang, P. Cui, J. Wang, X. Zhang, Y. Guan, W. Tan, G. Wu, H. Chen, Z. Bu, Susceptibility of ferrets, cats, dogs, and other domesticated animals to SARS-coronavirus 2, Science 368 (2020) 5.

[8] Q. Zhang, H. Zhang, K. Huang, Y. Yang, X. Hui, J. Gao, X. He, C. Li, W. Gong, Y. Zhang, C. Peng, X. Gao, H. Chen, Z. Zou, Z. Shi, M. Jin, SARS-CoV-2 neutralizing serum antibodies in cats: a serological investigation, bioRxiv (2020).

[9] P.J. Halfmann, M. Hatta, S. Chiba, T. Maemura, S. Fan, M. Takeda, N. Kinoshita, S.I. Hattori, Y. SakaiTagawa, K. Iwatsuki-Horimoto, M. Imai, Y. Kawaoka, Transmission of SARS-CoV-2 in Domestic Cats, N Engl J Med (2020).

[10] T.H.C. Sit, C.J. Brackman, S.M. Ip, K.W.S. Tam, P.Y.T. Law, E.M.W. To, V.Y.T. Yu, L.D. Sims, D.N.C. Tsang, D.K.W. Chu, R. Perera, L.L.M. Poon, M. Peiris, Infection of dogs with SARS-CoV-2, Nature (2020).

[11] S.F. Sia, L.M. Yan, A.W.H. Chin, K. Fung, K.T. Choy, A.Y.L. Wong, P. Kaewpreedee, R. Perera, L.L.M. Poon, J.M. Nicholls, M. Peiris, H.L. Yen, Pathogenesis and transmission of SARS-CoV-2 in golden hamsters, Nature (2020).

[12] W. Li, M.J. Moore, N. Vasilieva, J. Sui, S.K. Wong, M.A. Berne, M. Somasundaran, J.L. Sullivan, K. Luzuriaga, T.C. Greenough, H. Choe, M. Farzan, Angiotensin-converting enzyme 2 is a functional receptor for the SARS coronavirus, Nature 426 (2003) 5.

[13] M. Hoffmann, H. Kleine-Weber, S. Schroeder, N. Kruger, T. Herrler, S. Erichsen, T.S. Schiergens, G. Herrler, N.H. Wu, A. Nitsche, M.A. Muller, C. Drosten, S. Pohlmann, SARS-CoV-2 Cell Entry Depends on ACE2 and TMPRSS2 and Is Blocked by a Clinically Proven Protease Inhibitor, Cell 181 (2020) 271-280 $\mathrm{e} 278$.

[14] Y. Qiu, Y.B. Zhao, Q. Wang, J.Y. Li, Z.J. Zhou, C.H. Liao, X.Y. Ge, Predicting the angiotensin converting enzyme 2 (ACE2) utilizing capability as the receptor of SARS-CoV-2, Microbes Infect (2020).

[15] S. Kumar, G. Stecher, M. Li, C. Knyaz, K. Tamura, MEGA X: Molecular Evolutionary Genetics Analysis across Computing Platforms, Mol Biol Evol 35 (2018) 1547-1549.

[16] K. Katoh, D.M. Standley, MAFFT multiple sequence alignment software version 7: improvements in performance and usability, Mol Biol Evol 30 (2013) 772-780. 
[17] S. Lu, J. Wang, F. Chitsaz, M.K. Derbyshire, R.C. Geer, N.R. Gonzales, M. Gwadz, D.I. Hurwitz, G.H. Marchler, J.S. Song, N. Thanki, R.A. Yamashita, M. Yang, D. Zhang, C. Zheng, C.J. Lanczycki, A. Marchler-Bauer, CDD/SPARCLE: the conserved domain database in 2020, Nucleic Acids Res 48 (2020) D265-D268.

[18] I.A. Adzhubei, S. Schmidt, L. Peshkin, V.E. Ramensky, A. Gerasimova, P. Bork, A.S. Kondrashov, S.R. Sunyaev, A method and server for predicting damaging missense mutations, Nat Methods 7 (2010) 248-249.

[19] A. Waterhouse, M. Bertoni, S. Bienert, G. Studer, G. Tauriello, R. Gumienny, F.T. Heer, T.A.P. de Beer, C. Rempfer, L. Bordoli, R. Lepore, T. Schwede, SWISS-MODEL: homology modelling of protein structures and complexes, Nucleic Acids Res 46 (2018) W296-W303.

[20] X. Zhai, J. Sun, Z. Yan, J. Zhang, J. Zhao, Z. Zhao, Q. Gao, W.T. He, M. Veit, S. Su, Comparison of SARS-CoV-2 spike protein binding to ACE2 receptors from human, pets, farm animals, and putative intermediate hosts, J Virol (2020).

[21] K.D. Sonawane, S.S. Barale, M.J. Dhanavadeb, S.R. Waghmare, N.H. Nadaf, S.A. Kamble, A.A. Mohammed, A.M. Makandar, P.M. Fandilolu, A.S. Dound, N.M. Naik, , Homology modeling and docking studies of TMPRSS2 with experimentally known inhibitors Camostat mesylate, Nafamostat and Bromhexine hydrochloride to control SARS-Coronavirus-2, ChemRxiv (2020). 